\title{
From The Superparticle Path Integral To Superfield Theory
}

\author{
by \\ J.Grundberg \\ Department of Theoretical Physics \\ The Royal Institute of Technology \\ S-100 44 Stockholm \\ $S W E D E N$ \\ and \\ U.Lindström and H.Nordström \\ ITP \\ University of Stockholm \\ Vanadisvägen 9 \\ S-113 46 Stockholm \\ SWEDEN
}

\begin{abstract}
We investigate the hitherto unexplored relation between the superparticle path integral and superfield theory. Requiring that the path integral has the global symmetries of the classical action and obeys the natural composition property of path integrals, and also that the discretized action has the correct naive continuum limit, we find a viable discretization of the $(\mathrm{D}=3, \mathrm{~N}=2)$ free superparticle action. The resulting propagator is not the usual superfield one. We extend the discretization to include the coupling to an external gauge supermultiplet and use this to show the equivalence to superfield theory. This is possible since we are able to reformulate the superfield perturbation theory in terms of our new propagator.
\end{abstract}




\section{Introduction}

There have been numerous attempts to quantize both the massive and the massless superparticle [1]-[9]. Both the massless and massive models are invariant under a certain fermionic symmetry; the Siegel symmetry [2]. For the massive case quantization has been carried out both using BV-BRST methods [3] and using covariant methods [1]. In the massless case a covariant separation of the models first and second class constraints is not possible in general [4]. Attempts to circumvent this problem have been made using BVBRST methods [5] and using harmonic superspace methods [7]. Also noncovariant quantization has been described [8]. A constructive path integral quantization has, to our knowledge, only been attempted in [9], however.

We have given a brief report on the definition of the path integral in a letter [11]. Here we present the calculation in more detail along with an extensive discussion of the coupling to a background field.

Our construction starts from a set of (natural) requirements on the path integral; it should have the global symmetries of the classical action and it should obey the usual composition property of a path integral. In addition, we require the discretized action to have the correct naive continuum limit (i.e., assuming that the difference between the values of a function at time $t$ and $t+\varepsilon$ is of order $\varepsilon$ ). We find a discretization that complies with these demands and construct the propagator. This propagator differs from the usual field theory propagator as well as from the propagator derived in [9]. In particular, it contains an additional factor of an inverse momentum squared. This factor is dictated on dimensional grounds from the composition property and the dimension of the measure. Since it is unclear what physical meaning to ascribe to the superspace propagator for the free the-

ory, we study the coupling to a background gauge multiplet and find that the coupling is directly to the gauge potentials $\mathcal{A}$ rather than to the gauge prepotentials, which is the case for the superfield theory. We derive the Feynman rules and compare the perturbation expansion of the effective action to that of a (massless) chiral superfield coupled to a gauge super multiplet. We show equivalence explicitly to second order in the external field. In the process of doing this we show that it is possible to organize the superfield supergraph calculations in such a way that the propagator agrees with the one we derive from the superparticle. We have verified that the equivalence in fact holds to all orders in $\mathcal{A}$. 
As a further check on our methods, a similar calculation has been carried out comparing the superparticle in a light cone gauge and the light cone superfield theory [10].

Our analysis highlights that the path integral is a formal object which has to be given content by some evaluation prescription. This is particularly the case for fermionic variables. We also want to draw attention to the fact that the relation between the first quantized theory and the field theory is not always as simple as in the ordinary scalar field case. This is worth having in mind when trying to find a string field theory.

The organization of the article is as follows: In Sec.II we recapitulate the tensor calculus of [16] for constructing Siegel invariant actions in $D=3$ and present the action for the free superparticle as well as the action for a superparticle coupled to a gauge super multiplet. In Sec. III we exhibit a Legendre transform of the abovementioned actions and give the corresponding phase space actions which we subsequently use in the path integral. Sec. IV is devoted to the definition of the propagator via a discretization of the path integral. In Sec. V a survey of the supergraph rules for chiral fields coupled to a gauge prepotential superfield can be found along with the discussion of how to reorganize the perturbation expansion using the propagator derived from our particle path integral. A comparison of the superfield theory vertices to the interaction parts of our particle coupled to a gauge potential is then given in Sec. VI. Our conclusions constitute Sec. VII. In the Appendix we show the equivalence between the field theory and particle theory to all orders in the background field.

\section{Siegel Invariant Actions}

In [16] a tensor calculus for the Siegel-symmetry of $D=2$ and 3 superparticles is presented. It is based on a reformulation of the theories as supersymmetric $\sigma$-models that are invariant under local world-line superconformal transformations. The $N=2, D=3$ massive superparticle is described as follows:

The world line is extended to a $N=2$ world line in superspace with coordinates $\left\{z^{M}\right\}=(t, \eta, \bar{\eta})$ and the $D=3, N=2$ flat target superspace is coordinatized by $\left(X^{\mu}(z), \Theta_{\alpha}(z), \bar{\Theta}^{\alpha}(z)\right)$, a space-time vector and two spacetime spinors, all of which are world-line scalar fields. These fields are subject 
to the constraints

$$
\begin{aligned}
& \bar{D} X^{\mu}=-\frac{i}{2}(\bar{D} \bar{\Theta}) \Gamma^{\mu} \Theta \quad \text { and } \quad \text { c.c } \\
& \bar{D} \Theta=0 \text { and c.c., }
\end{aligned}
$$

where

$$
\left\{\Gamma^{\mu}, \Gamma^{\nu}\right\}=-2 \eta^{\mu \nu}, \quad \eta^{\mu \nu}=\operatorname{diag}(-++)
$$

and

$$
D \equiv \partial_{\eta}+i \bar{\eta} \partial_{t}, \quad \bar{D} \equiv-\partial_{\bar{\eta}}-i \eta \partial_{t}
$$

The constraints (四) lead to the following component expansion:

$$
\begin{aligned}
\Theta_{\alpha} \mid=\theta_{\alpha} & \bar{\Theta}^{\alpha} \mid=\bar{\theta}^{\alpha} \\
D \Theta_{\alpha} \mid=\lambda_{\alpha} & \bar{D} \bar{\Theta}^{\alpha} \mid=-\bar{\lambda}^{\alpha} \\
\frac{1}{2}[\bar{D}, D] \Theta_{\alpha} \mid=-i \dot{\theta}_{\alpha} & \frac{1}{2}[D, \bar{D}] \bar{\Theta}^{\alpha} \mid=-i \dot{\bar{\theta}}^{\alpha}
\end{aligned}
$$

and

$$
\begin{aligned}
X^{\mu} \mid & =x^{\mu} \\
D X^{\mu} \mid & =-\frac{i}{2} \bar{\theta} \Gamma^{\mu} \lambda \\
\bar{D} X^{\mu} \mid & =\frac{i}{2} \lambda \Gamma^{\mu} \theta,
\end{aligned}
$$

where $\mid$ denotes the $\eta=0$ projection. From (11) we also find the identity

$$
\bar{\lambda}^{\alpha} \lambda_{\beta}=\sqrt{-\pi^{2}} \delta_{\beta}^{\alpha}+\left(\Gamma^{\mu}\right)_{\beta}^{\alpha} \pi_{\mu}
$$

where

$$
\pi^{\mu} \equiv \dot{x}^{\mu}+\frac{i}{2}\left(\dot{\bar{\theta}} \Gamma^{\mu} \theta-\bar{\theta} \Gamma^{\mu} \dot{\theta}\right)
$$

The constraints are invariant under $N=2, \quad D=3$ space-time supersymmetry:

$$
\begin{aligned}
\delta X^{\mu} & =\frac{i}{2}\left(\bar{\rho} \Gamma^{\mu} \Theta-\bar{\Theta} \Gamma^{\mu} \rho\right) \\
\delta \Theta & =\rho, \quad \delta \bar{\Theta}=\bar{\rho}
\end{aligned}
$$


with $\rho$ and $\bar{\rho}$ two constant anticommuting space-time spinors. Furthermore there are also the local $N=2$ superconformal transformations

$$
\tilde{\delta} z^{M}=B \dot{z}^{M}+\frac{i}{2} \bar{D} B D z^{M}+\frac{i}{2} D B \bar{D} z^{M}
$$

with

$$
B|=-b(t), \quad D B|=2 i \bar{\varepsilon}(t), \quad \bar{D} B \mid=-2 i \varepsilon(t) .
$$

The action induced by (9) on the components in (4, 5) show that $b(t)$ is the parameter for infinitesimal reparametrizations while the identifications

$$
\varepsilon \equiv \frac{\bar{\lambda} \kappa}{\bar{\lambda} \lambda}
$$

yield

$$
\begin{aligned}
\delta_{\kappa} \theta_{\alpha} & =\frac{1}{2}\left(\delta_{\alpha}^{\beta}+\frac{\pi_{\alpha}^{\beta}}{\sqrt{\left(-\pi^{2}\right)}}\right) \kappa_{\beta} \\
\delta_{\kappa} \bar{\theta}^{\alpha} & =\frac{1}{2} \bar{\kappa}^{\beta}\left(\delta_{\beta}^{\alpha}+\frac{\pi_{\beta}^{\alpha}}{\sqrt{\left(-\pi^{2}\right)}}\right) \\
\delta_{\kappa} x^{\mu} & =\frac{i}{2}\left(\bar{\theta} \Gamma^{\mu} \delta_{\kappa} \theta-\delta_{\kappa} \bar{\theta} \Gamma^{\mu} \theta\right),
\end{aligned}
$$

i.e., the Siegel transformations, [2]. A covariantization of the theory with respect to the transformations (9) proceeds by introducing covariant derivatives on scalar fields as

$$
\nabla \Phi \equiv E^{-1} D \Phi, \quad \bar{\nabla} \Phi \equiv E^{-1} \bar{D} \Phi
$$

with

$$
E \equiv \sqrt{-\bar{D} \bar{\Theta} D \Theta}
$$

and covariant time derivative

$$
\nabla_{0} \Phi \equiv \frac{i}{2}\{\nabla, \bar{\nabla}\} \Phi .
$$

The world-line superconformal transformations are

$$
\delta(\nabla \Phi)=i L \nabla \Phi, \quad \delta(\bar{\nabla} \Phi)=-i L \bar{\nabla} \Phi
$$


where

$$
L=\bar{L} \equiv-\frac{1}{4}[D, \bar{D}] B
$$

A general Siegel-invariant action may now be written as

$$
S=\int_{0}^{1} d^{3} z \mathcal{L}\left(X, \Theta, \bar{\Theta}, \nabla \Theta, \bar{\nabla} \Theta, \nabla_{0} \Theta, \ldots\right) .
$$

The usual (second order) $N=2$ massive superparticle action [17 results from the choice

$$
\mathcal{L}=-\frac{m}{2} \bar{\Theta} \Theta .
$$

After integrating out the $\eta$-dependence,

$$
\begin{aligned}
S & =-\frac{m}{2} \int_{0}^{1} d^{3} z \bar{\Theta} \Theta \\
& =-m \int_{0}^{1} d \tau\left\{\sqrt{-\pi^{2}}+\frac{i}{2}(\dot{\bar{\theta}} \theta-\bar{\theta} \dot{\theta})\right\}
\end{aligned}
$$

Up to this point the description of the covariantization has followed Gauntlett [16] exactly. Now we adapt his results to our needs.

A special case of the general action (18) is

$$
\begin{aligned}
S & =\int_{0}^{1} d^{3} z \mathcal{L}(X, \Theta, \bar{\Theta}) \\
& =\frac{1}{2} \int_{0}^{1} d \tau[\bar{D}, D] \mathcal{L}(X, \Theta, \bar{\Theta}) \mid .
\end{aligned}
$$

Using the chain rule for differentiation, the definition of components (4.5) as well as the constraint relations (1), we expand the action (21) and find

$$
S=\int_{0}^{1} d \tau\left\{\frac{1}{2}\left[\lambda_{\alpha} \mathcal{D}^{\alpha}, \bar{\lambda}^{\beta} \overline{\mathcal{D}}_{\beta}\right] \mathcal{L}+i \dot{\bar{\theta}}^{\alpha} \overline{\mathcal{D}}_{\alpha} \mathcal{L}-i \dot{\theta}_{\alpha} \mathcal{D}^{\alpha} \mathcal{L}\right\}
$$


where

$$
\begin{aligned}
& \mathcal{D}^{\alpha} \equiv \frac{\partial}{\partial \theta_{\alpha}}-\frac{i}{2}\left(\bar{\theta} \Gamma^{\mu}\right)^{\alpha} \frac{\partial}{\partial x^{\mu}}, \\
& \overline{\mathcal{D}}_{\alpha} \equiv \frac{\partial}{\partial \bar{\theta}^{\alpha}}-\frac{i}{2}\left(\Gamma^{\mu} \theta\right)_{\alpha} \frac{\partial}{\partial x^{\mu}},
\end{aligned}
$$

which implies

$$
\left\{\overline{\mathcal{D}}_{\alpha}, \mathcal{D}^{\beta}\right\}=-i\left(\Gamma^{\mu}\right)_{\alpha}^{\beta} \frac{\partial}{\partial x^{\mu}} .
$$

Hence $\mathcal{D}_{\alpha}$ satisfy the global $D=3, \quad N=2$ supersymmetry algebra (in complex notation).

Using the identity (6), we finally have

$$
S=\int_{0}^{1} d \tau\left\{\sqrt{-\pi^{2}} \mathcal{D}^{\alpha} \overline{\mathcal{D}}_{\alpha} \mathcal{L}+\frac{1}{2} \pi_{\mu}\left[\mathcal{D}, \Gamma^{\mu} \overline{\mathcal{D}}\right] \mathcal{L}+i \dot{\bar{\theta}}^{\alpha} \overline{\mathcal{D}}_{\alpha} \mathcal{L}-i \dot{\theta}_{\alpha} \mathcal{D}^{\alpha} \mathcal{L}\right\} .
$$

If we let $\mathcal{L}=V(x, \theta, \bar{\theta})$ be a general real scalar superfield, this looks very much like a coupling of the particle to a gauge multiplet:

$$
S=\int_{0}^{1} d \tau\left[-\sqrt{-\pi^{2}} \mathcal{A}+\pi^{\mu} \mathcal{A}_{\mu}-i \dot{\theta}_{\alpha} \mathcal{A}^{\alpha}+i \dot{\bar{\theta}}^{\alpha} \overline{\mathcal{A}}_{\alpha}\right]
$$

where the gauge potentials are given in terms of the prepotential $V$ trough

$$
\begin{aligned}
& \overline{\mathcal{A}}_{\alpha}=\overline{\mathcal{D}}_{\alpha} V, \quad \mathcal{A}^{\alpha}=\mathcal{D}^{\alpha} V, \\
& \mathcal{A}^{\mu}=\frac{1}{2}\left[\mathcal{D}, \Gamma^{\mu} \overline{\mathcal{D}}\right] V, \quad \mathcal{A} \equiv-\mathcal{D}^{\alpha} \overline{\mathcal{D}}_{\alpha} V .
\end{aligned}
$$

That this describes the $N=2, D=3$ vector multiplet is perhaps most easily seen by dimensional reduction of the $N=1, D=4$ vector multiplet. $\mathcal{A}^{\mu}, \overline{\mathcal{A}}_{\alpha}$ and $\mathcal{A}^{\alpha}$ should certainly be present and $\mathcal{A}$ is the reduction of the fourth component of the vector potential. The constraints imply that they can be expressed in terms of a real scalar superfield as above. Gauge transformations take the form

$$
\delta V=i(\Lambda-\bar{\Lambda}), \quad \mathcal{D}^{\alpha} \bar{\Lambda}=\overline{\mathcal{D}}_{\alpha} \Lambda=0 .
$$


Using the identity

$$
\frac{d \Phi}{d \tau}=\pi^{\mu} \partial_{\mu} \Phi+\dot{\bar{\theta}}^{\alpha} \overline{\mathcal{D}}_{\alpha} \Phi+\dot{\theta}_{\alpha} \mathcal{D}^{\alpha} \Phi
$$

(valid for a general superfield $\Phi$ ), we find

$$
\delta S=\int_{0}^{1} d \tau \frac{d}{d \tau}(\Lambda-\bar{\Lambda}),
$$

so the action (25) transforms as it should under a gauge transformation.

\section{The phase space action}

As it stands, the action (25) has the disadvantage of involving a square root. We can get rid of this by making a Legendre transform with respect to $\pi_{\mu}$. Let

$$
\begin{aligned}
& L\left(\pi^{\mu}, \dot{\theta}, \dot{\bar{\theta}}, x, \theta, \bar{\theta}\right) \\
= & \left\{\sqrt{-\pi^{2}} \mathcal{D}^{\alpha} \overline{\mathcal{D}}_{\alpha}+\frac{1}{2}\left[\mathcal{D}^{\alpha},(\not \pi)_{\alpha}^{\beta} \overline{\mathcal{D}}_{\beta}\right]-i \dot{\bar{\theta}}^{\alpha} \overline{\mathcal{D}}_{\alpha}+i \dot{\theta}_{\alpha} \mathcal{D}^{\alpha}\right\} \mathcal{L}(x, \theta, \bar{\theta})
\end{aligned}
$$

and define

$$
\begin{aligned}
p_{\mu} & =\frac{\partial L}{\partial \pi^{\mu}} \\
H(p, \dot{\theta}, \dot{\bar{\theta}}, x, \theta, \bar{\theta}) & =p_{\mu} \pi^{\mu}-L .
\end{aligned}
$$

(The reader is invited to check that this is the usual transition to the hamiltonian for the case when $L$ does not depend explicitly on $\dot{\theta}$ and $\dot{\bar{\theta}}$.) Clearly terms in $L$ that are homogeneous of degree one in $\pi^{\mu}$ will not contribute to $H$.We thus find:

$$
H=\left(-i \dot{\bar{\theta}}^{\alpha} \overline{\mathcal{D}}_{\alpha}+i \dot{\theta}_{\alpha} \mathcal{D}^{\alpha}\right) \mathcal{L}
$$

Since

$$
p_{\mu}=\frac{\partial L}{\partial \pi^{\mu}}=\left(\frac{-\pi_{\mu}}{\sqrt{-\pi^{2}}} \mathcal{D}^{\alpha} \overline{\mathcal{D}}_{\alpha}+\frac{1}{2}\left[\mathcal{D}^{\alpha},\left(\Gamma_{\mu}\right)_{\alpha}^{\beta} \overline{\mathcal{D}}_{\beta}\right]\right) \mathcal{L}
$$


we also have a primary constraint

$$
\left[p_{\mu}-\frac{1}{2}\left[\mathcal{D}^{\alpha},\left(\Gamma_{\mu}\right)_{\alpha}^{\beta} \overline{\mathcal{D}}_{\beta}\right] \mathcal{L}\right]^{2}=-\left(\mathcal{D}^{\alpha} \overline{\mathcal{D}}_{\alpha} \mathcal{L}\right)^{2} .
$$

Incorporating this into the action with a Lagrange multiplier we get the phase space action

$$
\begin{aligned}
S^{P S}= & \int_{0}^{1} d \tau\left\{p_{\mu} \pi^{\mu}-e\left[\left[p_{\mu}-\frac{1}{2}\left[\mathcal{D}^{\alpha},\left(\Gamma_{\mu}\right)_{\alpha}^{\beta} \overline{\mathcal{D}}_{\beta}\right] \mathcal{L}\right]^{2}+\left(\mathcal{D}^{\alpha} \overline{\mathcal{D}}_{\alpha} \mathcal{L}\right)^{2}\right]\right. \\
& \left.+i \dot{\bar{\theta}}^{\alpha} \overline{\mathcal{D}}_{\alpha} \mathcal{L}-i \dot{\theta}_{\alpha} \mathcal{D}^{\alpha} \mathcal{L}\right\} .
\end{aligned}
$$

To get the final form of the action we write $\mathcal{L}=-\frac{m}{2} \bar{\theta} \theta+V$ and shift $p_{\mu}-\mathcal{A}_{\mu} \rightarrow$ $p_{\mu}$. The result is

$$
\begin{array}{r}
S=\int_{0}^{1} d \tau\left\{p_{\mu} \pi^{\mu}-e\left(p^{2}+(m+\mathcal{A})^{2}\right)-\frac{i}{2} m(\dot{\bar{\theta}} \theta-\dot{\theta} \bar{\theta})\right. \\
\left.+\pi_{\mu} \mathcal{A}^{\mu}+i \dot{\bar{\theta}} \overline{\mathcal{A}}_{\alpha}-i \theta_{\alpha} \mathcal{A}^{\alpha}\right\} .
\end{array}
$$

This should be compared to the $D=10$ superparticle in a super-Maxwell background discussed in [6].

\section{The Propagator}

The basic object in a path integral quantization is the propagator [13]. In the case of our free superparticle, it is symbolically given by

$$
K\left(x_{f}, \theta_{f}, \bar{\theta}_{f} ; x_{i}, \theta_{i}, \bar{\theta}_{i}\right)=\int D e D p D x D \theta D \bar{\theta} \exp \{i S\},
$$

where the action $S$ is (37) with the gauge potentials set to zero. We follow the usual treatment [2] in making the gauge choice

$$
\dot{e}=0 \quad \Rightarrow \quad e=T \text {. }
$$

The relation (38) is then replaced by

$$
K=\int_{0}^{\infty} d \tau G
$$


where

$$
G\left(x_{f}, \theta_{f}, \bar{\theta}_{f} ; x_{i}, \theta_{i}, \bar{\theta}_{i} ; T\right)=\int D p D x D \theta D \bar{\theta} \exp \{i S\}
$$

is a function of the final and initial superspace positions and

$$
S=\int_{0}^{T} d \tau\left\{p_{\mu}\left[\dot{x}^{\mu}+\frac{i}{2} \dot{\bar{\theta}} \Gamma^{\mu} \theta-\frac{i}{2} \bar{\theta} \Gamma^{\mu} \dot{\theta}\right]-\frac{i}{2} m(\dot{\bar{\theta}} \theta-\bar{\theta} \dot{\theta})-\left(p^{2}+m^{2}\right)\right\}
$$

It remains to give a meaning to this as yet symbolic expression for $G$. One way of defining a path integral is to discretize, i.e., to represent a path by the positions at a finite number of intermediate times, replace the action by a discretized version and integrate over the intermediate positions. A natural requirement is that the discretized action should have the original one as the (naive) limit as the number of time steps goes to infinity, but, as is well known, different discretizations complying with this condition can still give different results for the path integral. The root of the difficulty is that one is effectively summing over non-differentiable or even discontinuous paths, so the naive continuum limit is naive indeed. (This is the place where ordering problems enter the path integral scheme.) In addition to this difficulty, one also has to determine the measure for the integration over intermediate positions. We will base our treatment of these points on the symmetries of the problem and a general composition property of path integrals.

The composition property of path integrals is the intuitive rule that one can calculate the amplitude from $A$ to $C$ by multiplying the amplitude from $A$ to $B$ with the one from $B$ to $C$ and sum over the intermediate positions $B$ 13. In symbols

$$
G\left(3 ; 1 ; T_{1}+T_{2}\right)=\int d^{3} x_{2} d^{2} \theta_{2} d^{2} \bar{\theta}_{2} G\left(3 ; 2 ; T_{2}\right) G\left(2 ; 1 ; T_{1}\right)
$$

(where the arguments have been abbreviated, e.g., $\left(x_{3}, \theta_{3}, \bar{\theta}_{3}\right) \rightarrow 3$ et.c.). In formal derivations of path integrals this property expresses the completeness of intermediate states, from the intuitive point of view it is almost the defining property of path integrals. In perturbative calculations it is this property that gives the structure " propagator $\times$ verte $\times$ propagator $\times \ldots$..., a structure familiar from field theory and one we would also expect from first quantized treatment of the superparticle. Finally we note that the composition property 
(43) determines the dimension of $G$. Compared to the scalar particle path integral, the dimension of $G$ must be such that it cancels the dimension of the fermionic measure $d^{2} \theta d^{2} \bar{\theta}$.

For a free superparticle $G(2 ; 1 ; T)$ is to a large extent determined by the composition rule (43) and the symmetries of the problem. The latter, global $N=2$ Poincaré supersymmetry, implies that

$$
\begin{aligned}
G(2 ; 1 ; T)= & \int \frac{d^{3} p}{(2 \pi)^{3}} \tilde{G}\left(p, \theta_{2}-\theta_{1}, \bar{\theta}_{2}-\bar{\theta}_{1}\right) \\
\times & \exp \left\{i \left[p _ { \mu } \left(x_{2}^{\mu}-x_{1}^{\mu}+\frac{i}{4}\left(\bar{\theta}_{2}-\bar{\theta}_{1}\right) \Gamma^{\mu}\left(\theta_{2}+\theta_{1}\right)\right.\right.\right. \\
& \left.-\frac{i}{4}\left(\bar{\theta}_{2}+\bar{\theta}_{1}\right) \Gamma^{\mu}\left(\theta_{2}-\theta_{1}\right)\right)-\left(p^{2}+m^{2}\right) T \\
& \left.\left.-\frac{i m}{4}\left(\left(\bar{\theta}_{2}-\bar{\theta}_{1}\right)\left(\theta_{2}+\theta_{1}\right)-\left(\bar{\theta}_{2}+\bar{\theta}_{1}\right)\left(\theta_{2}-\theta_{1}\right)\right)\right]\right\},
\end{aligned}
$$

where we have also used the "dynamical" relation $i \partial_{T} G=\left(\left(-i \partial_{x}\right)^{2}+m^{2}\right) G$. The translational invariance of (44) is manifest, the supertranslational one can be expressed as

$$
\left(\mathcal{Q}_{2}^{\alpha}(1)+\mathcal{Q}_{1}^{\alpha}(-1)\right) G=\left(\overline{\mathcal{Q}}_{\alpha 2}(1)+\overline{\mathcal{Q}}_{\alpha 1}(-1)\right) G=0,
$$

where

$$
\begin{aligned}
\mathcal{Q}^{\alpha}(Z) & \equiv \frac{\partial}{\partial \theta_{\alpha}}+\frac{i}{2}\left(\bar{\theta} \Gamma^{\mu}\right)^{\alpha} \frac{\partial}{\partial x^{\mu}}+\frac{Z m}{2} \bar{\theta}^{\alpha} \\
\overline{\mathcal{Q}}_{\alpha}(Z) & \equiv \frac{\partial}{\partial \bar{\theta}^{\alpha}}+\frac{i}{2}\left(\Gamma^{\mu} \theta\right)_{\alpha} \frac{\partial}{\partial x^{\mu}}+\frac{m Z}{2} \theta_{\alpha} \\
\left\{\mathcal{Q}^{\alpha}(Z), \overline{\mathcal{Q}}_{\beta}(Z)\right\} & =i\left(\Gamma^{\mu}\right)_{\beta}^{\alpha} \frac{\partial}{\partial x^{\mu}}+m \delta_{\beta}^{\alpha} Z \\
\left\{\mathcal{Q}^{\alpha}(Z), \mathcal{Q}^{\beta}(Z)\right\} & =\left\{\overline{\mathcal{Q}}_{\alpha}(Z), \overline{\mathcal{Q}}_{\beta}(Z)\right\}=0
\end{aligned}
$$

are the generators of $N=2$ supersymmetry with central charge proportional to $Z$.

At this point we could try to impose the composition rule (43) to determine $\tilde{G}$. (We note in passing that for a scalar particle this would indeed give $\tilde{G}=1$.) The result would not be unique, however. Instead we note that the symmetries of the problem do not prevent us from imposing the antichirality condition

$$
\mathcal{D}_{2}^{\alpha}(1) G=0
$$


where

$$
\begin{aligned}
\mathcal{D}^{\alpha}(Z) & \equiv \frac{\partial}{\partial \theta_{\alpha}}-\frac{i}{2}\left(\bar{\theta} \Gamma^{\mu}\right) \frac{\partial}{\partial x^{\mu}}-\frac{Z m}{2} \bar{\theta}^{\alpha} \\
\overline{\mathcal{D}}_{\alpha}(Z) & \equiv \frac{\partial}{\partial \bar{\theta}^{\alpha}}-\frac{i}{2}\left(\Gamma^{\mu} \theta\right) \frac{\partial}{\partial x^{\mu}}-\frac{Z m}{2} \theta_{\alpha} \\
\left\{\mathcal{D}^{\alpha}(Z), \overline{\mathcal{D}}_{\beta}(Z)\right\} & =-i\left(\Gamma^{\mu}\right)_{\beta}^{\alpha} \frac{\partial}{\partial x^{\mu}}-Z m \delta_{\beta}^{\alpha} \\
\left\{\mathcal{D}^{\alpha}(Z), \mathcal{D}^{\beta}(Z)\right\} & =\left\{\overline{\mathcal{D}}_{\alpha}(Z), \overline{\mathcal{D}}_{\beta}(Z)\right\}=0
\end{aligned}
$$

are the $N=2$ supercovariant derivatives with central charge proportional to $Z$.

The antichirality condition (47) fixes the $\theta, \bar{\theta}$ dependence of $\tilde{G}$ and adding the composition condition (43) we find

$$
\begin{aligned}
G(2 ; 1 ; T) & =\int \frac{d^{3} p}{(2 \pi)^{3}} \frac{1}{p^{2}+m^{2}} \exp \left\{i \left(p _ { \mu } \left[x_{2}^{\mu}-x_{1}^{\mu}\right.\right.\right. \\
& \left.-\frac{i}{2} \bar{\theta}_{2} \Gamma^{\mu}\left(\theta_{2}-\theta_{1}\right)+\frac{i}{2}\left(\bar{\theta}_{2}-\bar{\theta}_{1}\right) \Gamma^{\mu} \theta_{1}\right] \\
& \left.\left.-\left(p^{2}+m^{2}\right) T-\frac{i}{2} m\left[\left(\bar{\theta}_{2}-\bar{\theta}_{1}\right) \theta_{1}-\bar{\theta}_{2}\left(\theta_{2}-\theta_{1}\right)\right]\right)\right\} .
\end{aligned}
$$

This is the final result for the free superparticle propagator. Apart from the symmetries, path integral properties were used in the derivation in the form of the dynamical relation $i \partial_{\tau} G=\left(\left(-i \partial_{x}\right)^{2}+m^{2}\right) G$ and the composition property (43).

We can now iterate (49) to build up a path integral. The result is

$$
\begin{aligned}
& G(f ; i ; T)= \\
& \lim _{N \rightarrow \infty} \int \prod_{k=1}^{N-1}\left(\frac{d^{3} p_{k} d^{3} x_{k} d^{2} \theta_{k} d^{2} \bar{\theta}_{k}}{(2 \pi)^{3}\left(p_{k}^{2}+m^{2}\right)}\right) \frac{d^{3} p_{N}}{(2 \pi)^{3}\left(p_{N}^{2}+m^{2}\right)} \exp \{i S\},
\end{aligned}
$$

where

$$
\begin{aligned}
S= & \sum_{k=1}^{N}\left(-\left(p_{k}^{2}+m^{2}\right) \varepsilon+p_{\mu}^{k} \pi_{k, k-1}^{\mu} \varepsilon\right. \\
& \left.-\frac{i}{2} m\left[\left(\bar{\theta}_{k}-\bar{\theta}_{k-1}\right) \theta_{k-1}-\bar{\theta}_{k}\left(\theta_{k}-\theta_{k-1}\right)\right]\right)
\end{aligned}
$$

and

$$
\pi_{k, k-1}^{\mu} \varepsilon \equiv x_{k}^{\mu}-x_{k-1}^{\mu}-\frac{i}{2} \bar{\theta}_{k} \Gamma^{\mu}\left(\theta_{k}-\theta_{k-1}\right)+\frac{i}{2}\left(\bar{\theta}_{k}-\bar{\theta}_{k-1}\right) \Gamma^{\mu} \theta_{k-1} .
$$


We have thus determined the measure and the discretized action. Note that the latter has the original Siegel-invariant action as the naive continuum limit. Note also the asymmetry in $\theta$ and $\bar{\theta}$. We remind the reader that a similar asymmetry exists in the path integral approach to fermion fields using coherent states [14].

The result (49) for the propagator was not quite unique. In addition to symmetries and general path integral ideas, (composition rule plus dynamical equation), we imposed an antichirality condition. It is clear that we could have used chirality instead, and the only difference would be $\bar{\theta} \leftrightarrow \theta$ everywhere. Are there other possibilities?

Some insight into this question is obtained by noting that the result for $G$ can be written

$$
\begin{aligned}
& G(f ; i ; T)= \\
& \frac{1}{2} \mathcal{D}_{f}^{\alpha} \mathcal{D}_{f}^{\beta} \overline{\mathcal{D}}_{\beta}^{i} \overline{\mathcal{D}}_{\alpha}^{i}\left[\delta^{4}\left(\theta_{f}-\theta_{i}\right) \int \frac{d^{3} p}{(2 \pi)^{3}} \frac{1}{p^{2}+m^{2}} e^{\left\{-i T\left(p^{2}+m^{2}\right)+i p_{\mu}\left(x_{f}^{\mu}-x_{i}^{\mu}\right)\right\}}\right],
\end{aligned}
$$

where we have suppressed the values of the central charge. Since $\left(\overline{\mathcal{D}}^{i}(-1)\right)^{2}$ acts on $\delta^{4}\left(\theta_{f}-\theta_{i}\right) \exp \left\{i p_{f}\left(x_{f}-x_{i}\right)\right\}$ we can replace it by $\left(\overline{\mathcal{D}}_{f}(1)\right)^{2}$, and, combining $\mathcal{D}^{2} \overline{\mathcal{D}}^{2}$ with the $\left(p^{2}+m^{2}\right)^{-1}$, we have the antichiral projection operator $\mathcal{D}^{2} \overline{\mathcal{D}}^{2} /\left(p^{2}+m^{2}\right)$ acting. Some thought shows that a projection operator acting on $\delta^{4}\left(\theta_{f}-\theta_{i}\right)$ is indeed what is needed to satisfy the composition property (43). In addition to the antichiral and chiral projection operators we may also consider the linear projector as a third and final independent projection operator. The discretized action corresponding to this alternative contains extra $\theta$-terms that vanish in the continuum limit. We will comment further on this in the conclusions.

As was mentioned in the introduction, another attempt to define the superparticle path integral by discretization has been made in [9]. Those authors proceed by writing down a discretization compatible with the global supersymmetry. Their discretization is the one that is obtained by using the "midpoint rule"

$$
\begin{aligned}
& x(t) \rightarrow \frac{x_{i+1}+x_{i}}{2} \\
& \dot{x}(t) \rightarrow \frac{x_{i+1}-x_{i}}{\varepsilon}
\end{aligned}
$$


for both bosonic and fermionic variables. In the notation of this paper it corresponds to having $\tilde{G}$ independent of $\theta, \bar{\theta}$. Such a discretization does not satisfy the composition property, but has the advantage of being always applicable. As is noted in [9], the result depends on whether the number of steps in the discretization is even or odd. The authors of [9] choose an odd number of steps to ensure an even number of integrals for each Lorentz component of $\theta$. Their final result for $K$ (denoted $G$ in [9]), for a massless superparticle is

$$
K=\mathcal{D}^{N^{\prime}} \int \frac{d^{D} p}{(2 \pi)^{D}} \frac{\exp \left\{i p\left(x_{f}-x_{i}\right)\right\}}{p^{2}} \delta^{N^{\prime}}\left(\theta_{f}-\theta_{i}\right)
$$

where $\mathcal{D}^{N^{\prime}}$ is the antisymmetrized product of all the covariant derivatives. This differs from our result in two respects, in the derivative structure and in the momentum dependence. Our result for $G$ implies that $K$ has $p^{4}$ in the denominator instead of the $p^{2}$ above. On the other hand the authors of 99 associate factors $p^{-2}$ with interaction over $\bar{\theta}_{k}, \theta_{k}$ in the discretization. Our objection to this approach is that it violates the basic composition rule of path integrals, and as a consequence it is harder to understand how to incorporate interactions. If this is done trough a discretization, one would certainly also need to use the propagator obtained with an even number of intermediate steps and thus obtain a perturbation theory with two kinds of propagators. A more techniqual objection is that to associate the $p^{-2}$ factors in the measure with the $\theta, \bar{\theta}$ integration rather than the $p$ integration seems unnatural to us. The discretization is a set of points in superspace $(x, \theta, \bar{\theta})$ with $p$ associated with the links between these points, and one would not expect these two kinds of variables to mix in the measure for a free particle.

What about the relation to superfield theory? The result for a scalar particle might lead one to expect the path integral propagator $K$ to be identical to the field theory one. This turns out not to be the case. Massless chiral superfield theory is described by the propagator

$$
\left\langle\bar{\Phi}\left(x_{f}, \theta_{f}, \bar{\theta}_{f}\right) \Phi\left(x_{i}, \theta_{i}, \bar{\theta}_{i}\right)\right\rangle=i \mathcal{D}_{f}^{2} \overline{\mathcal{D}}_{f}^{2}\left\{\int \frac{d^{3} p}{(2 \pi)^{2}} \frac{e^{i p\left(x_{f}-x_{i}\right)}}{p^{2}} \delta^{4}\left(\theta_{f}-\theta_{i}\right)\right\} .
$$

(It is customary to associate the derivatives with the vertices, but this is only a matter of book keeping.) The derivative structure is the same, the momentum dependence is not. It is however not a priori clear what $\langle\bar{\Phi} \Phi\rangle$ means 
in terms of propagating from one point in superspace to another, or, indeed, what that means in more physical terms. To make a meaningful comparison between the path integral and superfield theory, we have to consider quantities where we have better a priori reasons for thinking that the result should be the same. For this reason we turn to the calculation of the effective action induced by a superparticle in an external gauge field. As far as the particle is concerned we are then considering a closed loop and the question of the physical meaning of moving between points in superspace disappears.

\section{A Supergraph Survey}

As we saw in the previous section, our construction of the path integral for the superparticle does not result in the propagator that is ordinarily used in supergraph calculations. We had earlier noted that the superparticle couples to an external gauge field through the dimensionful gauge potentials. Superfields, on the other hand, couple through the dimensionless prepotential. It is conceivable that the differences in coupling and propagator in the two descriptions cancel and that the particle path integral and superfield description give the same results for physical quantities. In this section we review the superfield results we need to make a comparison, in particular we describe a way of rewriting the supergraph rules such that gauge potentials rather than prepotentials do enter the calculation. For further information about these topics we refer the reader to [15].

The superfield action for a massless chiral field coupled to an external electromagnetic gauge field is

$$
S=\int d^{3} x d^{2} \theta d^{2} \bar{\theta} \bar{\Phi} e^{-2 V} \Phi
$$

where $V$ is a dimensionless prepotential. Gauge transformations act on the fields as follows:

$$
\begin{aligned}
\Phi & \rightarrow e^{i \Lambda} \Phi, \quad \overline{\mathcal{D}} \Lambda=0 \\
\bar{\Phi} & \rightarrow \bar{\Phi} e^{-i \bar{\Lambda}}, \quad \mathcal{D} \bar{\Lambda}=0 \\
e^{-2 V} & \rightarrow e^{i \bar{\Lambda}} e^{-2 V} e^{-i \Lambda}
\end{aligned}
$$

The supergraph rules are obtained by expanding the action (57) in powers of $V$ to get the vertices, and by tying these together by the $\langle\bar{\Phi} \Phi\rangle$ propagator 
given in (56). The effective action is the sum of one loop graphs. We will not discuss the details of the calculation, but remind the reader that at the end of the day the result can be expressed in terms of gauge potentials and field strengths. This property can be made more manifest by reorganizing the calculation in a way similar to the "doubling" trick in $Q E D$. The essential idea is that the result of the one-loop calculation is the superdeterminant of the equations of motion operator. By considering the square of this operator instead one can show that the effective action $\Gamma$ can be written, (the notation $\mathcal{D}^{2} \overline{\mathcal{D}}^{2}$, et c., was introduced below (53)),

$$
\begin{aligned}
e^{i \Gamma=} & \exp \left\{-i \int d^{3} x d^{2} \bar{\theta} \frac{1}{2} \frac{\delta}{\delta j} \mathcal{D}^{2}\left(\bar{\nabla}^{2}-\overline{\mathcal{D}}^{2}\right) \frac{\delta}{\delta j}\right\} \\
& \times\left.\exp \left\{\frac{i}{2} \int d^{3} x d^{2} \bar{\theta} j \partial^{-2} j\right\}\right|_{j=0},
\end{aligned}
$$

where

$$
\begin{aligned}
\bar{\nabla}_{\alpha} & \equiv \overline{\mathcal{D}}_{\alpha}-2\left(\overline{\mathcal{D}}_{\alpha} V\right) \\
\frac{\delta j(x, \bar{\theta})}{\delta j\left(x^{\prime}, \bar{\theta}^{\prime}\right)} & =\mathcal{D}^{2} \delta^{3}\left(x-x^{\prime}\right) \delta^{4}\left(\theta-\theta^{\prime}\right) .
\end{aligned}
$$

The result is that the effective action is obtained by evaluating graphs consisting of closed strings

$$
\ldots\left[-i \mathcal{D}^{2}\left(\bar{\nabla}^{2}-\overline{\mathcal{D}}^{2}\right)\right]_{k}\left(\frac{-i \delta^{4}\left(\theta_{k}-\theta_{k-1}\right)}{p^{2}}\right)\left[-i \mathcal{D}^{2}\left(\bar{\nabla}^{2}-\overline{\mathcal{D}}^{2}\right)\right]_{k-1} \ldots
$$

At this point we depart from the standard treatment and note that once vertex $k$ has operated on $\delta^{4}\left(\theta_{k}-\theta_{k-1}\right)$, the expression is chiral as a function of $\theta_{k}$. We can thus, without changing anything, insert the chiral projection operator and thus replace the propagator $\frac{-i \delta^{4}\left(\theta_{k}-\theta_{k-1}\right)}{p^{2}}$ by the propagator we derived from the path integral. This can be done for all propagators in the loop. Thus we have shown that the effective action corresponding to the coupling of a chiral field to an external $U(1)$ gauge field can be calculated using our path integral derived propagator and using $i \mathcal{D}^{2}\left(\bar{\nabla}^{2}-\overline{\mathcal{D}}^{2}\right)$ as vertex. In the next section we will show that something equivalent to this vertex can be derived from the particle path integral point of view, and thus show that the particle path integral and the field theory calculations indeed give the same result. 


\section{First Quantized Feynman Rules}

In the previous section we showed that superfield supergraph calculations can be organized in such a way that the propagator becomes the one we derived by path integral quantization of a superparticle. This section will be devoted to the vertex.

The contribution to the effective action of the $k$-th vertex is, according to the superfield theory, given by

$$
\int d^{3} x_{k} d^{2} \theta_{k} d^{2} \bar{\theta}_{k} K_{0}(k+1 ; k) \frac{(-i)}{2} \mathcal{D}_{k}^{\alpha} \mathcal{D}_{k}^{\beta}\left(\bar{\nabla}_{\beta}^{k} \bar{\nabla}_{\alpha}^{k}-\overline{\mathcal{D}}_{\beta}^{k} \overline{\mathcal{D}}_{\alpha}^{k}\right) K_{0}(k ; k-1),
$$

where $K_{0}$ is the free propagator

$$
K_{0}(f ; i)=-\frac{i}{2} \mathcal{D}_{f}^{\alpha} \mathcal{D}_{f}^{\beta} \overline{\mathcal{D}}_{\beta}^{i} \overline{\mathcal{D}}_{\alpha}^{i}\left[\int \frac{d^{3} p}{(2 \pi)^{3}} \frac{\delta^{4}\left(\theta_{f}-\theta_{i}\right)}{p^{4}} e^{i p \cdot\left(x_{f}-x_{i}\right)}\right]
$$

This can equivalently be written as

$$
\begin{aligned}
& \int d^{3} x_{k} d^{2} \theta_{k} d^{2} \bar{\theta}_{k} K_{0}(k+1 ; k) i\left\{2 i \check{\partial}_{\mu}^{k}\left(\Gamma^{\mu}\right)_{\alpha}^{\beta}\left(\mathcal{D}^{\alpha} \overline{\mathcal{D}}_{\beta} V\right)\right. \\
& \left.+\left(\mathcal{D}^{\alpha} \mathcal{D}^{\beta} \overline{\mathcal{D}}_{\beta} \overline{\mathcal{D}}_{\alpha} V\right)-2 \mathcal{D}^{\alpha} \mathcal{D}^{\beta}\left(\overline{\mathcal{D}}_{\beta} V \overline{\mathcal{D}}_{\alpha} V\right)\right\} K_{0}(k, k-1),
\end{aligned}
$$

where we have integrated by parts and made use of the chirality properties

of $K_{0}$ and the definition of $\bar{\nabla}_{\alpha}$. Note that the vertex is asymmetric, as was the propagator, and note also that it contains both terms linear in $V$ and quadratic in $V$ ("seagull" terms). The interaction lagrangian (26) contains only part of the quadratic term. We will construct the discretized version of the interaction in such a way that it gives the linear term. That second order perturbation theory automatically reproduces the "seagull" terms is then a nontrivial check on the construction. Ultimately this goes reflects the gauge invariance, of course.

The problem of discretizing the path integral has two parts: The action and the measure. We begin with the latter. Recall that for the free particle the measure is given by

$$
\prod_{k=1}^{N-1} \frac{d^{3} x_{k} d^{3} p_{k} d^{2} \theta_{k} d^{2} \bar{\theta}_{k}}{(2 \pi)^{3}\left(p_{k}^{2}+m^{2}\right)} \frac{d^{3} p_{N}}{(2 \pi)^{3}\left(p_{N}^{2}+m^{2}\right)} .
$$


When interactions are present the mass enters in the action in the combination $m-\mathcal{D}^{\alpha} \overline{\mathcal{D}}_{\alpha} V$. This suggests that the measure should be given by

$$
\prod_{k=1}^{N-1} \frac{d^{3} x_{k} d^{3} p_{k} d^{2} \theta_{k} d^{2} \bar{\theta}_{k}}{(2 \pi)^{3}\left(p_{k}^{2}+\left(\mathcal{D}^{\alpha} \overline{\mathcal{D}}_{\alpha} V\right)_{k-1}^{2}\right)} \frac{d^{3} p_{N}}{(2 \pi)^{3}\left(p_{N}^{2}+\left(\mathcal{D}^{\alpha} \overline{\mathcal{D}}_{\alpha} V\right)_{N-1}^{2}\right)}
$$

Here, as well as in what follows, we have restricted ourselves to the $m=0$ case.

Next we turn to the discretization of the interaction part of the action. In the corresponding treatment of a non-relativistic particle in an external field this is the place where the midpoint rule (54) plays an important role. With the midpoint rule a discretized action exists which is consistent with gauge invariance and has the correct naive continuum limit, without it one has to sacifice the continuum limit to preserve gauge invariance. We already noted that the vertex we want to recover looks asymmetric and so does the propagator we constructed. With hindsight we sacrifice the midpoint construction and pay the price. We suggest the following discretization:

$$
\begin{aligned}
S= & \sum_{i} \varepsilon\left\{\pi_{i+1, i}^{\mu}\left(\Gamma_{\mu}\right)_{\alpha}^{\beta}\left(\mathcal{D}^{\alpha} \overline{\mathcal{D}}_{\beta} V\right)_{i}-\left(\mathcal{D}^{\alpha} \overline{\mathcal{D}}_{\alpha} V\right)_{i}^{2}\right. \\
& \left.+2 i \frac{\left(\bar{\theta}_{i+1}-\bar{\theta}_{i}\right)^{\alpha}}{\varepsilon}\left(\overline{\mathcal{D}}_{\alpha} V\right)_{i}+\left(\mathcal{D}^{\beta} \mathcal{D}^{\alpha} \overline{\mathcal{D}}_{\alpha} \overline{\mathcal{D}}_{\beta} V\right)_{i}\right\} .
\end{aligned}
$$

Before showing that this reproduces the linear term of the vertex, we discuss the continuum limit and gauge invariance.

The naive continuum limit is

$$
S=\int_{0}^{T} d t\left\{\pi_{\alpha}^{\beta} \mathcal{D}^{\alpha} \overline{\mathcal{D}}_{\beta} V+2 i \dot{\bar{\theta}}^{\alpha} \overline{\mathcal{D}}_{\alpha} V-\left(\mathcal{D}^{\alpha} \overline{\mathcal{D}}_{\alpha} V\right)^{2}+\left(\mathcal{D}^{\beta} \mathcal{D}^{\alpha} \overline{\mathcal{D}}_{\alpha} \overline{\mathcal{D}}_{\beta} V\right)\right\}
$$

The first three terms are reasonable, they just differ from the interaction terms in (37) by a total derivative. The last term is the price we pay for the asymmetry. If we make a gauge transformation (28), $\delta V=i(\bar{\Lambda}-\Lambda)$, the result should be a function of the endpoints. We obtain

$$
\delta S=\int_{0}^{T} d t\left\{-2 \pi^{\mu} \partial_{\mu} \bar{\Lambda}-2 \dot{\bar{\theta}}^{\alpha} \overline{\mathcal{D}}_{\alpha} \bar{\Lambda}-2 i \partial^{2} \bar{\Lambda}\right\},
$$

reminiscent of the Ito formula [12]

$$
f(b)-f(a)=\int_{a}^{b} d t \frac{d x}{d t} \frac{d f}{d x}+\int_{a}^{b} d t \frac{1}{2}\left(\frac{d^{2} f}{d x^{2}}\right),
$$


an equation which is valid in a (Wiener) path integral if the integrals in the equation are understood as asymmetric Riemann sums:

$$
\int_{a}^{b} d t \frac{d x}{d t} \frac{d f}{d x}=\lim _{N \rightarrow \infty} \sum_{k=1}^{N} \varepsilon \frac{\left(x_{k}-x_{k-1}\right)}{\varepsilon} \frac{d f}{d x}\left(x_{k-1}\right) .
$$

After this discussion of the discretized action, we now proceed to show that it does reproduce the linear terms of the field theory vertex. The path integral for the effective action is given by

$$
2 i \Gamma=\lim _{N \rightarrow \infty} \int_{0}^{\infty} \frac{d T}{T} \int \prod_{k=1}^{N} \frac{d^{3} x_{k} d^{3} p_{k} d^{2} \theta_{k} d^{2} \bar{\theta}_{k}}{(2 \pi)^{3}\left(p_{k}^{2}+\left(\mathcal{D}^{\alpha} \overline{\mathcal{D}}_{\alpha} V\right)_{k-1}^{2}\right)} e^{i\left(S_{0}+S_{I}\right)}
$$

where $S_{0}$ is the discretized free action (51) and $S_{I}$ is the discretized interaction (67). Note that the measure contains the same number of $(x, \theta, \bar{\theta})$ and $p$ integrations, in contrast to the situation for the propagator. This is because we are calculating a loop, so we have to integrate over the initial = final point as well. That we are calculating a loop is also reflected in the $T^{-1}$-factor in the $T$-integral [20].

Just as for the non-relativistic particle [13], the effect of the interaction is reduced to calculations of certain transition elements and we wish to relate these to derivatives of the propagator. For the linear order of the $k$ th term in the discretized interaction we need to calculate expressions of the form 


$$
\begin{aligned}
& \int d^{3} x_{k+1} d^{4} \theta_{k+1} d^{3} x_{k} d^{4} \theta_{k} G(x, \theta, \bar{\theta} ; k+1) G(k+1 ; k ; \varepsilon) \\
& i \varepsilon\left\{\pi_{k+1, k}^{\mu}\left(\Gamma_{\mu}\right)_{\alpha}^{\beta}\left(\mathcal{D}^{\alpha} \overline{\mathcal{D}}_{\beta} V\right)_{k}+2 i \frac{\left(\bar{\theta}_{k+1}-\bar{\theta}_{k}\right)^{\alpha}}{\varepsilon}\left(\overline{\mathcal{D}}_{\alpha} V\right)_{k}\right. \\
+ & \left.\left(\mathcal{D}^{\alpha} \mathcal{D}^{\beta} \overline{\mathcal{D}}_{\beta} \overline{\mathcal{D}}_{\alpha} V\right)_{k}\right\} G(k ; y, \eta, \bar{\eta}),
\end{aligned}
$$

where $G$ denotes the free propagator (49). Using the explicit expression (49) for $G(k+1 ; k ; \varepsilon)$ it is easy to show that

$$
\begin{aligned}
G(k+1 ; k ; \varepsilon) \pi_{k+1, k}^{\mu} & =2 G(k+1 ; k ; \varepsilon) i \partial_{k}^{\mu}-\frac{2 i}{\varepsilon} \int \frac{d^{3} p}{(2 \pi)^{3}} \frac{p^{\mu}}{p^{4}} e^{i \varepsilon\left\{p \cdot \pi_{k+1, k}-p^{2}\right\}} \\
G(k+1 ; k ; \varepsilon) \frac{\left(\bar{\theta}_{k+1}-\bar{\theta}_{k}\right)^{\alpha}}{\varepsilon} & =\frac{-1}{\varepsilon} \mathcal{D}_{k}^{\beta} \int \frac{d^{3} p}{(2 \pi)^{3}} \frac{\not p_{\beta}^{\alpha}}{p^{4}} e^{i \varepsilon\left\{p \cdot \pi_{k+1, k}-p^{2}\right\} .}
\end{aligned}
$$

We insert these expressions in $(73)$ and integrate the $\mathcal{D}_{k}^{\beta}$ term by parts. Since $G(k ; y, \eta, \bar{\eta})$ is antichiral, only the term where $\mathcal{D}^{\beta}$ acts on $\overline{\mathcal{D}}_{\alpha} V$ survives and cancels the second term in the expression for $G(k+1 ; k ; \varepsilon) \pi^{\mu}$. The factor $\varepsilon$ becomes the measure in the integral over the time at which the integration acts, and thus the net result is

$$
\begin{aligned}
\int d t \int d^{3} x_{k} d^{2} \theta_{k} d^{2} \bar{\theta}_{k} \quad & G(x, \theta, \bar{\theta} ; k) i\left[2 i{\stackrel{\llcorner}{\partial_{k}^{\mu}}}_{k}\left(\Gamma_{\mu}\right)_{\alpha}^{\beta}\left(\mathcal{D}^{\alpha} \overline{\mathcal{D}}_{\beta} V\right)\right. \\
& \left.+\left(\mathcal{D}^{\alpha} \mathcal{D}^{\beta} \overline{\mathcal{D}}_{\beta} \overline{\mathcal{D}}_{\alpha} V\right)\right] G(k ; y, \eta, \bar{\eta})
\end{aligned}
$$

We have reproduced the linear part of the vertex. In the above expression the propagators are $G$ rather than $K_{0}$, but this difference disappears once we integrate over $T$ and the times at which the interactions occur: The particle path integral gives us the parametric representation of amplitudes. Before we proceed with a discussion of the "seagull" terms we emphasize that the discontinuous character of the paths in superspace make objects like $\pi^{\mu}$ and $\dot{\bar{\theta}}$ divergent. It is only the sum of the $\pi^{\mu} A_{\mu}$ and $\dot{\bar{\theta}}^{\alpha} \bar{A}_{\alpha}$ terms that is finite. Note also that the antichiral character of the propagator was important for the cancellation.

The quadratic terms in the vertex arise in second order perturbation theory. We get contributions both from expanding $e^{i S_{I}}$ and from the measure. From the former we get quadratic terms of the form (vertex at $j) \times(v e r t e x$ at 
$k), j \neq k$. These are treated as above. In addition we get a "contact" term

$$
\begin{aligned}
& \frac{(i \varepsilon)^{2}}{2 !} \int d^{3} x_{k+1} d^{4} \theta_{k+1} d^{3} x_{k} d^{4} \theta_{k} G(x, \theta, \bar{\theta} ; k+1) G(k+1 ; k ; \varepsilon) \\
& \left\{\pi_{k+1, k}^{\mu}\left(\Gamma_{\mu}\right)_{\alpha}^{\beta}\left(\mathcal{D}^{\alpha} \overline{\mathcal{D}}_{\beta} V\right)_{k}+2 i \frac{\left(\bar{\theta}_{k+1}-\bar{\theta}_{k}\right)^{\alpha}}{\varepsilon}\left(\overline{\mathcal{D}}_{\alpha} V\right)_{k}\right. \\
+ & \left.\left(\mathcal{D}^{\alpha} \mathcal{D}^{\beta} \overline{\mathcal{D}}_{\beta} \overline{\mathcal{D}}_{\alpha} V\right)_{k}\right\}^{2} G(k ; y, \eta, \bar{\eta}) .
\end{aligned}
$$

For the terms containing $\mathcal{D}^{2} \overline{\mathcal{D}}^{2} V$ we can use the same trick as for the linear terms in the vertex. $\mathcal{D}^{2} \overline{\mathcal{D}}^{2} V$ is antichiral, so the integration by parts is not affected and the result for these terms is

$$
\begin{array}{rl}
\frac{(i \varepsilon)^{2}}{2 !} \int d^{3} x_{k} d^{2} \theta_{k} d^{2} \bar{\theta}_{k} & G(x, \theta, \bar{\theta} ; k)\left[2 \cdot 2 i \check{\partial}_{k}^{\mu}\left(\Gamma_{\mu}\right)_{\alpha}^{\beta}\left(\mathcal{D}^{\alpha} \overline{\mathcal{D}}_{\beta} V\right)_{k}\left(\mathcal{D}^{\rho} \mathcal{D}^{\sigma} \overline{\mathcal{D}}_{\sigma} \overline{\mathcal{D}}_{\rho} V\right)_{k}\right. \\
& \left.+\left(\mathcal{D}^{\alpha} \mathcal{D}^{\beta} \overline{\mathcal{D}}_{\beta} \overline{\mathcal{D}}_{\alpha} V\right)_{k}^{2}\right] G(k ; y, \eta, \bar{\eta})
\end{array}
$$

The rest of the calculation is no different in principle from the linear calculation, but messier. One writes down expressions for $\not_{k+1, k \alpha}^{\beta} \not_{k+1, k \rho}^{\delta}$ et.c., similar to (74) and integrates by parts. Just as for the non-relativistic particle, one gets some extra terms in addition to the square of the linear contribution. The total result,including the terms in (77), is

$$
\begin{aligned}
& \frac{i^{2}}{2 !} \varepsilon^{2} \int d^{3} x_{k} d^{2} \theta_{k} d^{2} \bar{\theta}_{k} G(x, \theta, \bar{\theta} ; k)\left\{\left[(2 i \grave{\phi})_{\alpha}^{\beta}(2 i \grave{\partial})_{\gamma}^{\delta}\left(\mathcal{D}^{\alpha} \overline{\mathcal{D}}_{\beta} V\right)_{k}\left(\mathcal{D}^{\gamma} \overline{\mathcal{D}}_{\delta} V\right)_{k}\right.\right. \\
& \left.+2(2 i \not{\partial})_{\alpha}^{\beta}\left(\mathcal{D}^{\alpha} \overline{\mathcal{D}}_{\beta} V\right)_{k}\left(\mathcal{D}^{\gamma} \mathcal{D}^{\delta} \overline{\mathcal{D}}_{\delta} \overline{\mathcal{D}}_{\gamma} V\right)_{k}+\left(\mathcal{D}^{\alpha} \mathcal{D}^{\beta} \overline{\mathcal{D}}_{\beta} \overline{\mathcal{D}}_{\alpha} V\right)_{k}^{2}\right] \\
& \left.+\frac{2 i}{\varepsilon}\left[2 \mathcal{D}^{\alpha} \mathcal{D}^{\beta}\left(\overline{\mathcal{D}}_{\beta} V \overline{\mathcal{D}}_{\alpha} V\right)_{k}-\left(\mathcal{D}^{\alpha} \overline{\mathcal{D}}_{\alpha} V\right)_{k}^{2}\right]\right\} G(k ; y, \eta, \bar{\eta}) \\
& +\int d^{3} x_{k+1} d^{4} \theta_{k+1} d^{3} x_{k} d^{4} \theta_{k} \frac{d^{3} p}{(2 \pi)^{3}} G(x, \theta, \bar{\theta} ; k+1) \\
& \times e^{i \varepsilon\left(p \cdot \pi_{k+1, k}-p^{2}\right)} \frac{\left(\mathcal{D}^{\alpha} \overline{\mathcal{D}}_{\alpha} V\right)^{2}}{p^{4}} G(k ; y, \eta, \bar{\eta}) .
\end{aligned}
$$

The $\mathcal{O}\left(\varepsilon^{2}\right)$-terms combine with the (vertex $\left.j\right) \times($ vertex $k)$ terms to give the second order contribution of the linear terms in the field theory vertex. $\left(\varepsilon^{2}\right.$ is the measure for integration over two independent interaction times.) The $\mathcal{O}(\varepsilon)$ term, combined with the $(\mathcal{D} \overline{\mathcal{D}} V)^{2}$ term in the action, reproduces the 
field theory "seagull" terms and the $\mathcal{O}\left(\varepsilon^{0}\right)$ term cancels the contribution from the measure to this order. This completes the demonstration that the second order perturbation theory based on the discretized interaction (67) coincides with the superfield theory. The full equivalence is shown in the appendix .

\section{Conclusions}

As mentioned in the introduction, the massive Siegel invariant superparticle has been quantized (in various dimensions) using BRST-methods [3], as well as canonical ones [1], (without calculating the propagator however). No difficulties of principle were encountered. For the massless case, the canonical procedure is faced with the difficulty that a covariant separation of first and second class constraints is impossible [4. This has led to quantization using Batalin-Vilkovisky type Lagrangian BRST methods [5]. These constructions involve an infinite tower of ghosts. Our construction would seem to circumvent both these difficulties. We have ignored the Siegel invariance, although the (anti)chirality with respect to the endpoints may be viewed as a remnant of this symmetry. The issue of first and second class constraints never arises, the construction involves no ghosts, and the limit $m \rightarrow 0$ seems unproblematic. Our treatment of the path integral may seem to be particular to $D=3, N=2$, but it is clear that this construction of the propagator works just as well for the $D=4, N=1$ massless superparticle. What about other cases?

The assumptions in this article are, (explicitly), the composition property (43) and, (implicitly), that the exponent in the infinitesimal propagator should look "reasonable" as a discretization of the action. In a conventional treatment of the path integral with gauge fixing and ghosts, a modified version of (43) involving also ghost coordinates should be satisfied, since this is essentially the completeness property of intermediate states. Truncating to $\left(x^{\mu}, \theta, \bar{\theta}\right)$ is thus an assumption that the ghost coordinates decouple, which is reasonable. The second assumption is more questionable. Gauge fixing would certainly modify the action and with the gauge condition $\dot{\theta}=0$ [18], the $\theta$-dependent part of the propagator would be $\delta^{N}\left(\theta_{f}-\theta_{i}\right)$, (which certainly satisfies the composition property). The role of the ghosts is to remove un-

physical states from this, i.e., to project onto an irreducible representation 
of supersymmetry. This is precisely what the projection operator in our construction accomplishes. (C.f. the situation in string theory with ghosts versus the Brink-Olive projection operator [19]). It thus seems that the existence of a projection operator that projects onto an irreducible representation of supersymmetry is the basic requirement in our construction.

We also want to draw attention to an alternative way of viewing our results. Starting from the three basic ingredients, the dynamical relation $i \partial_{\tau} G=\left(\left(-i \partial_{x}\right)^{2}+m^{2}\right) G$, the composition property (43) and supersymmetry, we find a limited number of possible definitions of the path integral for a supersymmetric object satisfying $p^{2}+m^{2}=0$. All involve projection operators acting on $\delta\left(\theta_{f}-\theta_{i}\right)$. If we confine ourselves to the usual independent set of projection operators [15], they give path integrals which can be viewed as arising from the usual superparticle action. In this sense we derive that action from the path integral!

Note added: After the completion of this work we became aware of a related paper [21]. Starting from the superfield theory, these authors derive a path itegral representation of the Greens functions which involves the superparticle action. They give no explicit definition of the path integral, however.

Acknowledgements: We thank H.Hansson, A.Karlhede, M.Roček and W.Siegel for numerous discussions on the subject of this article. We also thank R.Marnelius for drawing our attention to 21 


\section{Appendix}

In this appendix we prove that the equivalence between superfield theory and superparticle path integral that we demonstrated to second order in Section VI holds to all orders.

Consider the contribution of the $k$-th term in the discretized free action (51) and the interaction (67). It is given by

$$
\begin{gathered}
\int \frac{d^{3} p}{(2 \pi)^{3}} d^{3} x_{k} d^{4} \theta_{k} \frac{e^{i \varepsilon\left(p \cdot \pi_{k+1, k}-p^{2}\right)}}{p^{2}+\left(\mathcal{D}^{\alpha} \overline{\mathcal{D}}_{\alpha} V\right)_{k}^{2}} \times \\
\exp \left\{i \varepsilon \left[\pi_{k+1, k}^{\mu}\left(\Gamma_{\mu}\right)_{\alpha}^{\beta}\left(\mathcal{D}^{\alpha} \overline{\mathcal{D}}_{\beta} V\right)_{k}-\left(\mathcal{D}^{\alpha} \overline{\mathcal{D}}_{\alpha} V\right)_{k}^{2}\right.\right. \\
\left.\left.+2 i \frac{\left(\bar{\theta}_{k+1}-\bar{\theta}_{k}\right)^{\alpha}}{\varepsilon}\left(\overline{\mathcal{D}}_{\alpha} V\right)_{k}+\left(\mathcal{D}^{\beta} \mathcal{D}^{\alpha} \overline{\mathcal{D}}_{\alpha} \overline{\mathcal{D}}_{\beta} V\right)_{k}\right]\right\} F\left(x_{k}, \theta_{k}, \bar{\theta}_{k}\right),
\end{gathered}
$$

where $F(k)$ is the contribution from the terms $1, \ldots, k-1$. The entire $k$ dependence of $F$ is carried by $\pi_{k, k-1}$, and thus

$$
\mathcal{D}^{\alpha} F(k)=0
$$

Expressing $\pi_{k+1, k}$ and $\bar{\theta}_{k+1}-\bar{\theta}_{k}$ in the interaction as suitable derivatives we can rewrite (A1) as follows:

$$
\begin{gathered}
\int \frac{d^{3} p}{(2 \pi)^{3}} d^{3} x_{k} d^{4} \theta_{k} F(k) \frac{\exp \left\{i \varepsilon\left[-p^{2}-\left(\mathcal{D}^{\alpha} \overline{\mathcal{D}}_{\alpha} V\right)_{k}^{2}+\left(\mathcal{D}^{\beta} \mathcal{D}^{\alpha} \overline{\mathcal{D}}_{\alpha} \overline{\mathcal{D}}_{\beta} V\right)_{k}\right]\right\}}{p^{2}+\left(\mathcal{D}^{\alpha} \overline{\mathcal{D}}_{\alpha} V\right)_{k}^{2}} \times \\
\exp \left\{i \varepsilon \frac{1}{i \varepsilon}\left(\mathcal{D} \Gamma^{\mu} \overline{\mathcal{D}}\right)_{k} \frac{\partial}{\partial p^{\mu}}\right\} \hat{\mathcal{O}} e^{i \varepsilon p \cdot \pi_{k+1, k}},
\end{gathered}
$$

where

$$
\hat{\mathcal{O}}=1-2 \frac{\not p_{\alpha}^{\beta}}{p^{2}}\left(\overline{\mathcal{D}}_{\beta} V\right)_{k} \mathcal{D}_{k}^{\alpha}+2 \frac{\not p_{\alpha}^{\beta}}{p^{2}}\left(\overline{\mathcal{D}}_{\beta} V\right)_{k} \frac{p_{\gamma}^{\delta}}{p^{2}}\left(\overline{\mathcal{D}}_{\delta} V\right)_{k} \mathcal{D}_{k}^{\gamma} \mathcal{D}_{k}^{\alpha}
$$

Integrating $\frac{\partial}{\partial p}$ in (A3) by parts gives an operator that shifts the variable $p$ by $(\mathcal{D} \Gamma \overline{\mathcal{D}})$, (alternatively, we could have made the shift already in $(\mathrm{A} 1)$ ). Inserting also $1=\hat{\mathcal{O}} \hat{\mathcal{O}}^{-1}$, we get

$$
\int \frac{d^{3} p}{(2 \pi)^{3}} d^{3} x_{k} d^{4} \theta_{k} F(k) \hat{\mathcal{O}} \hat{\mathcal{O}}^{-1} \frac{1}{A} \exp \{-i \varepsilon B\} \hat{\mathcal{O}} e^{i \varepsilon p \cdot \pi_{k+1, k}},
$$


where

$$
\begin{gathered}
A \equiv\left(p-(\mathcal{D} \Gamma \overline{\mathcal{D}} V)_{k}\right)^{2}+\left(\mathcal{D}^{\alpha} \overline{\mathcal{D}}_{\alpha} V\right)_{k}^{2} \\
=p^{2}+2 \mathcal{D}^{\alpha} \mathcal{D}^{\beta}\left(\overline{\mathcal{D}}_{\beta} V \overline{\mathcal{D}}_{\alpha} V\right)_{k}+4\left(\overline{\mathcal{D}}_{\alpha} V\right)\left(\mathcal{D}^{\alpha} \mathcal{D}^{\beta} \overline{\mathcal{D}}_{\beta} V\right)_{k}-2(\mathcal{D} \not p \overline{\mathcal{D}} V)_{k} \\
B \equiv A-\left(\mathcal{D}^{\alpha} \mathcal{D}^{\beta} \overline{\mathcal{D}}_{\beta} \overline{\mathcal{D}}_{\alpha} V\right)_{k} .
\end{gathered}
$$

We will in fact never need an explicit expression for $\hat{\mathcal{O}}^{-1}$, but it is clear that it can be constructed in perturbation theory. Next we use the identity

$$
\hat{\mathcal{O}}^{-1} f(A, B) \hat{\mathcal{O}}=f\left(\hat{\mathcal{O}}^{-1} A \hat{\mathcal{O}}, \hat{\mathcal{O}}^{-1} B \hat{\mathcal{O}}\right),
$$

and note that antichiral objects commute with $\hat{\mathcal{O}}$. Thus

$$
\begin{gathered}
\hat{\mathcal{O}}^{-1} A \hat{\mathcal{O}}=A+\hat{\mathcal{O}}^{-1}[A, \hat{\mathcal{O}}] \\
=A+\hat{\mathcal{O}}^{-1}\left[-2(\mathcal{D} \not p \overline{\mathcal{D}} V)_{k}+4\left(\overline{\mathcal{D}}_{\alpha} V\right)_{k}\left(\mathcal{D}^{\alpha} \mathcal{D}^{\beta} \overline{\mathcal{D}}_{\beta} V\right)_{k}, \hat{\mathcal{O}}\right] \\
\hat{\mathcal{O}}^{-1} B \hat{\mathcal{O}}=\hat{\mathcal{O}}^{-1} A \hat{\mathcal{O}}-\left(\mathcal{D}^{\alpha} \mathcal{D}^{\beta} \overline{\mathcal{D}}_{\beta} \overline{\mathcal{D}}_{\alpha} V\right)_{k} .
\end{gathered}
$$

What saves us from the need of knowing $\hat{\mathcal{O}}^{-1}$ is the fact that

$$
\left[-2(\mathcal{D} \not p \overline{\mathcal{D}} V)_{k}+4\left(\overline{\mathcal{D}}_{\alpha} V\right)_{k}\left(\mathcal{D}^{\alpha} \mathcal{D}^{\beta} \overline{\mathcal{D}}_{\beta} V\right)_{k}, \hat{\mathcal{O}}\right]=-\hat{\mathcal{O}} 4\left(\overline{\mathcal{D}}_{\alpha} V\right)_{k}\left(\mathcal{D}^{\alpha} \mathcal{D}^{\beta} \overline{\mathcal{D}}_{\beta} V\right)_{k}
$$

Proof: The $R H S$ can be written as

$$
\left[4\left(\overline{\mathcal{D}}_{\alpha} V\right)_{k}\left(\mathcal{D}^{\alpha} \mathcal{D}^{\beta} \overline{\mathcal{D}}_{\beta} V\right)_{k}, \hat{\mathcal{O}}\right]-4\left(\overline{\mathcal{D}}_{\alpha} V\right)_{k}\left(\mathcal{D}^{\alpha} \mathcal{D}^{\beta} \overline{\mathcal{D}}_{\beta} V\right)_{k} \hat{\mathcal{O}},
$$

so we only have to show that

$$
\left[2(\mathcal{D} \not \overline{\mathcal{D}} V)_{k}+4\left(\overline{\mathcal{D}}_{\alpha} V\right)_{k}\left(\mathcal{D}^{\alpha} \mathcal{D}^{\beta} \overline{\mathcal{D}}_{\beta} V\right)_{k}, \hat{\mathcal{O}}\right]=-4\left(\overline{\mathcal{D}}_{\alpha} V\right)_{k}\left(\mathcal{D}^{\alpha} \mathcal{D}^{\beta} \overline{\mathcal{D}}_{\beta} V\right)_{k} \hat{\mathcal{O}}
$$

which is trivial to verify.

Note that the second derivative term in $\hat{\mathcal{O}}$ doesn't contribute to the $R H S$ because it is multiplied by $(\overline{\mathcal{D}} V)^{3}=0$.

Returning to (A8) and using (A6), we see that (A5) can be written

$$
\begin{gathered}
\int \frac{d^{3} p}{(2 \pi)^{3}} d^{3} x_{k} d^{4} \theta_{k} F(k) \hat{\mathcal{O}} \times \\
\frac{\exp \left\{-i \varepsilon\left(-p \cdot \pi_{k+1, k}+p^{2}-2(\mathcal{D} \not p \overline{\mathcal{D}} V)_{k}+2 \mathcal{D}^{\alpha} \mathcal{D}^{\beta}\left(\overline{\mathcal{D}}_{\beta} V \overline{\mathcal{D}}_{\alpha} V\right)_{k}-\left(\mathcal{D}^{\alpha} \mathcal{D}^{\beta} \overline{\mathcal{D}}_{\beta} \overline{\mathcal{D}}_{\alpha} V\right)_{k}\right)\right\}}{p^{2}-2(\mathcal{D} \not p \overline{\mathcal{D}} V)_{k}+2 \mathcal{D}^{\alpha} \mathcal{D}^{\beta}\left(\overline{\mathcal{D}}_{\beta} V \overline{\mathcal{D}}_{\alpha} V\right)_{k}} .
\end{gathered}
$$


Finally we integrate the $\mathcal{D}$ 's in $\hat{\mathcal{O}}$ by parts. Using that $F(k)$ is antichiral, we find that the result replaces the denominator in (A12) by $p^{2}$. The final result is thus

$$
\begin{gathered}
\int \frac{d^{3} p}{(2 \pi)^{3}} d^{3} x_{k} d^{4} \theta_{k} \frac{e^{i \varepsilon\left(p \cdot \pi_{k+1, k}-p^{2}\right)}}{p^{2}} \exp \left\{i \varepsilon \left[2(\mathcal{D} \not p \overline{\mathcal{D}} V)_{k}-\right.\right. \\
\left.\left.-2 \mathcal{D}^{\alpha} \mathcal{D}^{\beta}\left(\overline{\mathcal{D}}_{\beta} V \overline{\mathcal{D}}_{\alpha} V\right)_{k}+\left(\mathcal{D}^{\alpha} \mathcal{D}^{\beta} \overline{\mathcal{D}}_{\beta} \overline{\mathcal{D}}_{\alpha} V\right)_{k}\right]\right\} F(k) .
\end{gathered}
$$

This shows shows agreement with the superfield theory to all orders in perturbation theory, (c.f. (64)). 


\section{References}

[1] J.M. Evans, Nucl. Phys., B331 (1990) 711.

[2] W. Siegel, Phys. Lett., 128B (1983) 397.

[3] M.B. Green and C.M. Hull, Contribution to "Strings 89", World Scientific 1989, 478.

[4] I. Bengtsson, and M. Cederwall, Göteborg Preprint 84-21 June 1984.

[5] U.Lindström, M.Roček, W. Siegel, P. van Nieuwenhuizen and A.E. van de Ven, Phys. Lett. 224B (1989) 285.

U.Lindström, M.Roček, W. Siegel, P. van Nieuwenhuizen and A.E. van de Ven, Phys. Lett. 228B (1989) 53.

U.Lindström, M.Roček, W. Siegel, P. van Nieuwenhuizen and A.E. van de Ven, Journ. Math. Phys. 31 (1990) 1761.

[6] M.Roček, W. Siegel, P. van Nieuwenhuizen and A.E. van de Ven, Phys. Lett. 227B (1989) 87.

[7] E. Sokatchev, Phys. Lett. 169B (1986) 209; Class. Quantum Grav. 4 (1987)237.

L. Brink, M. Hennaux and C. Teitelboim Nucl. Phys. B293 (1987) 505.

E. Nissimov, A. Pacheva and S. Solomon, Nucl. Phys. B296 (1988) 462; B299 (1988) 183.

[8] J.M. Evans, Class. Quantum Grav., 7 (1990) 699.

[9] A. Miković and W. Siegel, Phys. Lett. 240B (1990) 363.

[10] H.Nordström, "A light cone superparticle-superfield theory relation",(in preparation).

[11] J. Grundberg, U. Lindström and H. Nordström, "Discretization of the Superparticle Path Integral", University of Stockholm preprint, USITP92-11, October 1992, hep-th/9211024 
[12] L.S. Schulman, "Techniques and Applications of Path Integration", J. Wiley and sons, New York 1981.

[13] R.P. Feynman and A.R. Hibbs, "Quantum Mechanics and Path Integrals", McGraw-Hill, New York 1965.

[14] Y. Ohnuki and K. Kashiwa, Prog. Theor. Phys. 60 (1978) 548.

[15] S.J. Gates, M.T. Grisaru M. Roček and W. Siegel, "SUPERSPACE", Benjamin/Cummings, Reading 1983.

[16] J.P. Gauntlett, Phys. Lett. 272B (1991) 25.

[17] J.P. Gauntlett and C.F. Yastremiz, Class. Quantum Grav. 7 (1990) 2089.

[18] W. Siegel, Contribution to "Strings 89", World Scientific 1989, 211.

[19] M.B. Green, J.H. Schwarz and E. Witten, "Superstring Theory", Cambridge University Press, Cambridge 1987.

[20] A.M. Polyakov, "Gauge Fields and Strings", Harwood, 1987.

[21] E.S. Fradkin and SH.M. Svartsman, Mod. Phys. Lett. A6, (1991) 1977. 